\title{
Interpreter Glove With Gesture Recognition Ability For Dumb And Deaf Ones
}

\author{
1: Suryabhan Shaw \\ Electronics and Communication \\ Engineering \\ Bengal College of Engineering \\ Durgapur, India \\ sbshaw6668@gmail.com
}

\author{
2: Archana Kumari \\ Electronics and Communication \\ Engineering \\ Bengal College of Engineering \\ Durgapur, India \\ archanaarchu683@gmail.com
}

\author{
3: Samir Basak \\ (ISBN- 978-1-4673-6217-7/13) \\ Electronics and Communication \\ Engineering \\ Bengal College of Engineering \\ Durgapur, India \\ samirbasak69@gmail.com
}

\begin{abstract}
With the advent of wearable technology, it is now possible to implement numerous and extremely creative ideas to serve humanity in unprecedented ways. Thus inspired, we have developed a smart system which would be more beneficial for deaf and dumb ones. The primary goal of this paper is to design and implement a low cost wired interactive glove, interfaced with Arduino UNO, with a high degree of accuracy for gesture recognition. The glove maps the orientation of fingers with the help of flex sensors. The data is then transmitted to the smartphone with the help of Bluetooth module connected with arduino UNO. As, they have the ability to see they do read and write. The system is modeled for the differently abled section of the society to help convert sign language to more human understandable and efficient form such as interpretation or understandable voice/speech. As nowadays, smartphones are playing a vital role in our lifestyle. Thus inspired, we had developed an app which will interpret on behalf of hearing and speech impaired ones. It will vocalize the statement they write and do converts speech to text which they can read. If they fall in some trouble, they can crave for help just by making fist or using a hotkey integrated in the app which will make sound of siren. If in some bad day they have an accident, the smartphone finds an accidental shake and the helping message with the location will be sent to registered mobile number and the same message will be sent to ambulance and an automatic call will go for emergency and proper confirmation. This device will bridge the gap in communication and helps them to grow their career.
\end{abstract}

Keywords- Gesture recognition, data glove, data extraction method, voice interpretation, Save Our Souls (SOS), speech to text, text to speech.

\section{IntRoduction (HEADING 1)}

When human talks with one another, they communicate not just through their speech but also through their gestures. Gestures might either complement one's speech or totally substitute it especially in the case of hearing and speech impaired people. However, such differently abled person often has trouble communicating other people and find themselves in embarrassing situations. This is because different gestures might be used by different people to convey the same message. Hence, we have an intelligent system comprising a wired glove interfaced with Arduino UNO which can be customized to the gestures that a person is comfortable with and translate them into textual messages or speech.
Moreover, the above can be expanded to implement general purpose gesture recognition specifically modeled as a tool for bomb diffusing or for robotic arm, where it's risky to get closer to the bomb which can be diffused from far away with the help of long radio frequencies or can work as a prosthetic hand. Later in this paper we will propose the hardware modifications required to achieve this ambitious goal.

\section{LITERATURE REVIEW}

Various techniques have been employed in the recent past to achieve the objectives outlined in Section I. These include visual recognition techniques using image processing which, however, come with their own limitations [1]. Skin color detection, through a popular strategy used in computer vision-based algorithms, is sensitive to lighting conditions [2]. Moreover, a flexible and progressively adapting model for skin color recognition is a challenging task [1]. Besides, motion cues limit the gesturer to a stationary background [3].

The concept of wired gloves has also been used by researchers and developers in the recent past. Flex sensors and linear sensors together with backpropagation (BP) algorithm were proposed in [4]. However, a difficulty faced by the gesturer wearing such a glove in the restriction he feels while wearing it. Flex sensors were used in a data glove that used as an alternative to speech which they can't be able to produce.

\section{HARDWARE COMPONENTS AND THEORETICAL} BACKGROUND

\section{A. Flex Sensors}

A flex sensor comprises a substrate, conductor means and connector means. The substrate is formed of a deflectable and electrically insulating material. The conductor means is adhered to the substrate in a preselected pattern. The conductor means is formed of an electrically conductive ink which predictably changes electrical resistance upon deflection of the substrate between a first configuration and a second configuration. The connector means is associated with the conductor means for interconnection to external electrical components [5]. 
Signed letters are determined using flex sensor on each finger. The flex sensors change their resistance based on the amount of bend in the sensor as shown in figure. As a variable printed resistor, the flex sensor achieves great form factor on a thin. flexible substrate. When sensor placed in gloves is bent, it produces a resistance output correlated to the bend radius-the smaller the radius, the higher the resistance value. They require a 5-volt input and output between 0 and $5 \mathrm{~V}$. The sensors are connected to the device via three pin connectors (ground, live, and output). In device, sensors are activated in sleep mode. It enables them to power down mode when not in use. By voltage divider rule, output voltage is determined and given $\mathrm{V}$ out $=\mathrm{V}$ in $*$ $\mathrm{R} 1 /(\mathrm{R} 1+\mathrm{R} 2)$, where $\mathrm{R} 1$ is the other input resistor to the non-inverting terminal [6].

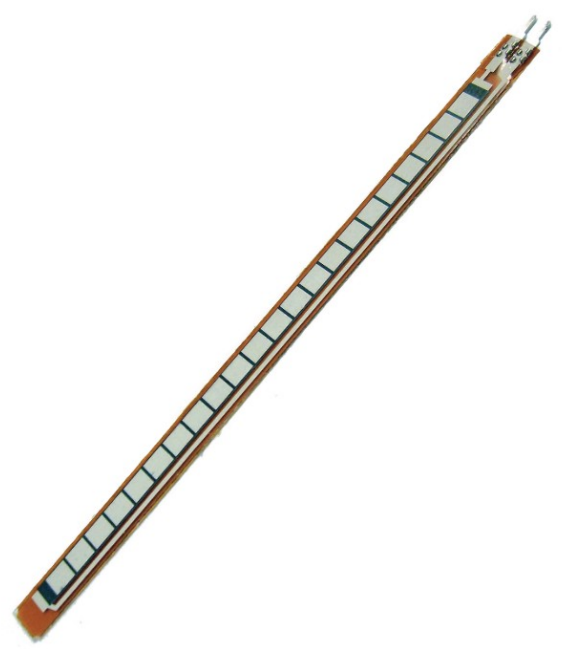

Figure 1: A sample of flex sensor

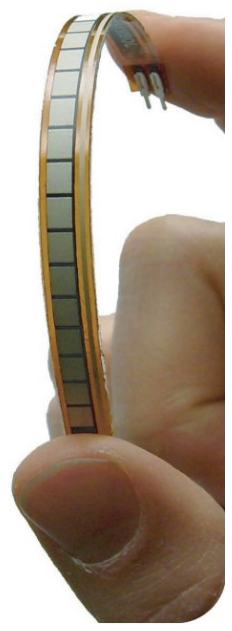

CHARACTERISTICS:
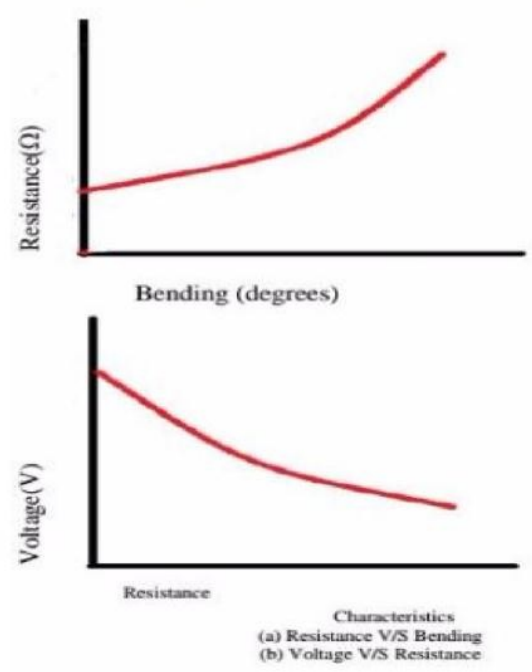

Figure 2: Characteristics of flex sensor

\section{B. Bluetooth Module (HC-05)}

HC-05 module is an easy to use Bluetooth SPP (Serial Port Protocol) module, designed for transparent wireless serial connection setup.

Serial port Bluetooth module is fully qualified Bluetooth V2.0+EDR (Enhanced Data Rate) 3Mbps Modulation with complete $2.4 \mathrm{GHz}$ radio trans receiver and baseband. It uses CSR Bluecore 04-External single chip Bluetooth system with CMOS technology and with AFH (Adaptive Frequency Hopping Feature). It has the footprint as small as $12.7 \mathrm{mmx} 27 \mathrm{~mm}$. Hope it will simplify your overall design/development cycle.
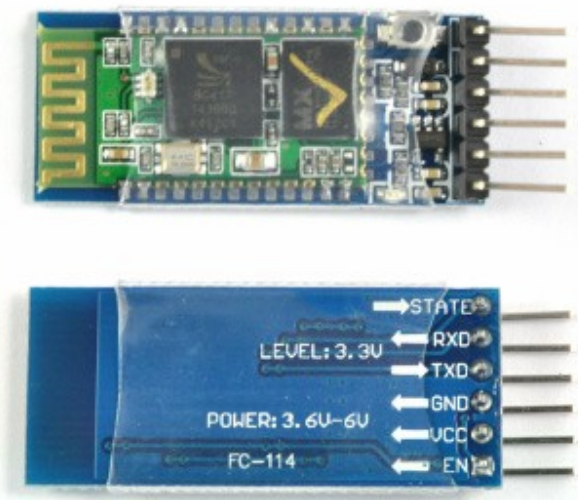

Figure 3: A sample of Bluetooth Module (HC-05)

\section{Arduino UNO}

The Arduino Uno is a microcontroller board based on the ATmega328P microcontroller. It has 14 digital input/output pins (of which 6 can be used as PWM outputs), 6 analogue inputs, a $16 \mathrm{MHz}$ quartz crystal, a USB connection, a power jack, an ICSP header and a reset button. Microcontroller is the heart of the device. It stores the required data and make use of it whenever the person uses the device. This device helps deaf and dumb person to announce their requirement. Arduino programs may be written in any programming language with a compiler that produces binary machine code. The Arduino project provides the Arduino integrated development environment (IDE), which is a cross-platform application written in the programming language Java. The Arduino IDE supports the languages $\mathrm{C}$ and $\mathrm{C}++$ using special rules to organize code. All output signals generated from flex sensors are in analogue form and these signals need to be digitized. The microcontroller digitizes all analogue signals from the sensors for sensor signal selection. It supports both serial and parallel communication facilities [6].

\section{Android App}

Developed an app containing following features with their functions: -

a) Bluetooth: To connect it with Bluetooth module connected with Arduino UNO which will transmit the data of flex sensors to the smartphone.

b) Text to speech: To covert the text to speech, which will make the deaf and dumb ones to communicate verbally. They just need to type what they want to say, even the app does have the feature to convey the local language which will make easy for them to communicate with others verbally.

c) Speech to text: To convert the speech to text, which the common or normal people will speak, and the 


\section{ISSN NO: 2350-1146 I.F-5.11}

app type the same as normal people speaks even in local language too.

d) Emergency Call button: It will be triggered when the smartphone finds an accidental shake and the helping message with the exact location will be sent to the family or concern ones and the same message will be sent to ambulance and an automatic call will be triggered for the assurance of the emergency.

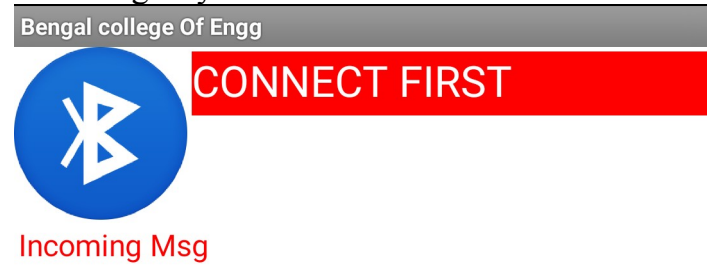

Speak

Hint for TextBox1

\section{Design and Developed By SuryaBhan Shaw}

Listen

Emergency Call with SMS ones can fold their fingers with some combinations. The combination patterns are as follows: -

- $1=1$

- $2=3$

- $3=7$

- $4=15$

- $5=31$

By observing above patterns, we can represent the same by the following general equation: -

Number of combinations $=\left(2^{\wedge} n\right)-1$, where $n$ stands for the number of fingers.

The resistance of the flex sensor changes and can request for the basic needs. To communicate with others verbally they need to type on the app as they have the ability to see, they can read or write on the app. As they write on the app, it will convert text to speech on click of speak button. And if the normal or common ones speaks something it do has the feature to convert speech to text which the dumb and deaf ones read from the app, which will make them easy to communicate with normal ones. If someday, they have an accident and the smartphone finds an accidental shake it will ask "Are you alright?". If got answer "Yes", then no message or call will be triggered. But if finds any other response either "Yes" or no response, it will trigger the helping message with location to the family or concerned ones. And again, a message with same location will be sent to the ambulance and an automatic call will go for the further assurance of emergency.

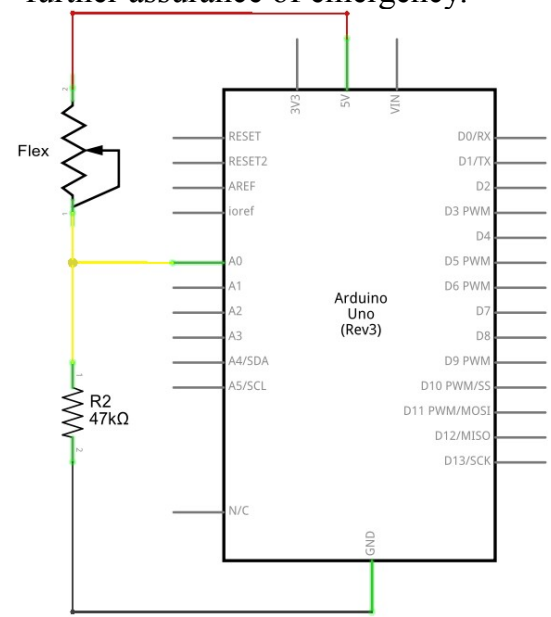

Figure 5: Schematic circuit [7]

The glove's purpose in this project is to capture the hand gestures of the user which the system intends to convert to sound signals. The glove has five flex sensors attached to the back of each of the five fingers and three contact sensors, two between index finger and middle finger and one on thumb. These sensors are connected to the analog inputs of the Arduino UNO which has a voltage divider circuit and a Bluetooth module is connected to the Arduino UNO to transmit the data from flex sensors.

An app is developed which will interpret on the behalf of dumb and the deaf ones. As it has a Bluetooth which will be connected to the Bluetooth module, and the dumb and deaf 


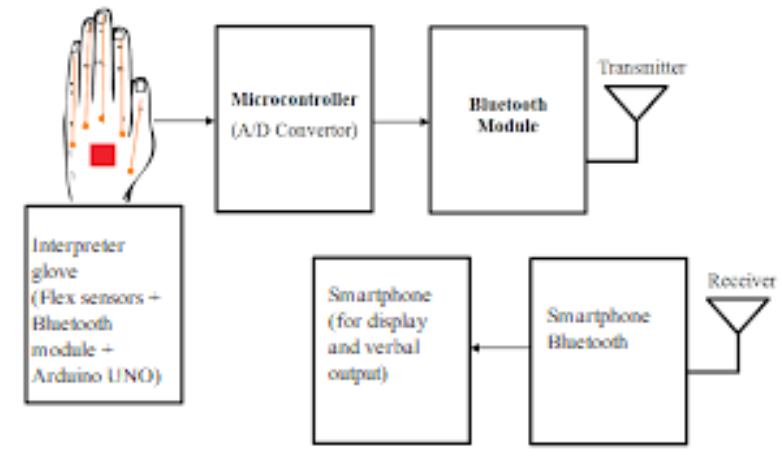

Figure 6: Flow Chart of the working model

OUTPUT

https://drive.google.com/open?

id=1LNF9Cpodbc35f1_BEqoH8VIP-Kzh519I

\section{RESULT}

- The deaf and dumb people utilize these gloves to change over the signs performed by them into speech.

- From the accommodation of simple flex sensors, a user can interact with others in more comfortable and simpler way. This makes it possible for the users to communicate within their community as well as with other people and they can carry on with a normal life.

- The end product will have an inexpensive and simple configuration making it simple for user to work with [6].

\section{FUTURE SCOPE}

- This system has been implemented on windows platform but it can be extended to other platforms.

- The delay required between two hand signs for proper functioning of system can be removed to make system more user friendly.
- This can be expanded for the robotic arm or can be modelled for bomb diffusion without risking their life.

\section{CODE}

https://rive.google.com/file/d/1PM6IiM0v7kHgvpsMvz0vXT6dte $\mathrm{UBo} 4 \mathrm{BD} /$ view? usp=drivesdk

\section{CONCLUSION}

Sign language is a method used for communication by disabled person. Here we are converting sign language into text and speech so that communication is not limited between them only, utilizing data gloves communication barrier between two different communities is eliminated. Using data gloves disabled person can also grow in their carrier and makes nation grow as percentage of disabled person are millions in count. Making their future better, making nation better.

\section{REFERENCES}

[1] P.Garg, N. Aggarwal and S. Sofat, "Vision based hand gesture recognition," World Academy of Science,Engineering and Technology, vol. 3, 2009.

[2] V. Pavlovic, R. Sharma and T. S. Huang, "Visual interpretation of hand gestures for human-computer interaction: a review," IEEE Transactions on Pattern Analysis and Machine Intelligence, vol. 19, no. 7, pp.677692, July 1997.

[3] P. Halarankar, S. Shah, H. Shah, H. Shah and J. Shah "Gesture recognition technology: a review," International Journal of Engineering Science and Technology (IJEST), vol. 4, no 11, 2012.

[4] C. S. Fahn and H Sun, "Development of a sensory data glove using neural-network-based calibration, "Corrections of IC-AT 2000 Proceeding, pp 239-245, 2000.

[5] C. S. Ingulkar and A. N. Gaikwad, "Hand data glove: a wearable real time device for human computer interaction," International Journal of Science and Engineering, vol. 1, pp-99-104, 2013.

[6] Abhishek Soni, Abhishek Kardak, Gokul K, "Missing sense a smart glove for sign to speech/text conversion," International Journal of Multidisciplinary Research and Development, vol. 3, pp-327-329, 2016.

[7] https://learn.sparkfun.com/tutorials/flex-sensor-hookup-guide/all 